\title{
The connection between absorptive capacity and open innovation in managerial perspective
}

\author{
Cristina Feniser ${ }^{1, *}$, Florin Lungu ${ }^{1}$, and Javier Bilbao ${ }^{2}$ \\ ${ }^{1}$ Department of Management and Economic Engineering Department, Faculty of Machine Building, \\ Technical University of Cluj-Napoca, Romania \\ ${ }^{2}$ Applied Mathematics Department, Engineering School, University of the Basque Country, \\ UPV/EHU, Spain
}

\begin{abstract}
The growing number of research dedicated to open innovation has determined a growth of company's interest towards external sources of knowledge and of the interactions with these resources in order to obtain an added value. Absorptive capacity, together with the outside-in dimension of open innovation represent interconnected concepts. The present study examines their connection both in theory and in their representation in SMEs managers' perception. The study that forms the base of the research is a survey conducted on companies' managers who worked on open innovation projects. Although few studies dealing with the subject existed up until now, the vision of the managers was chosen to be examined due to the impact of a managerial decision over a company's ability to learn from an exterior environment. In the future, open innovation is thought to be integrated in the management of innovation, this being the reason we view it as of utmost importance for the deepening of the managerial perspective regarding its influences and component elements.
\end{abstract}

\section{Open Innovation and absorptive capacity: literature overview}

Open Innovation and the capacity of absorption are both very well-known notions in contemporary literature dedicated to the management of innovation, but the connection between them have been systematically treated very few times until now. The growing number of research dedicated to open innovation has determined a growth of company's interest towards external sources of knowledge and of the interactions with these resources in order to obtain an added value. Chesbrough (2003) describes open innovation as " $a$ model that assumes that companies can and should use external ideas as well as internal ideas, and internal and external path towards the market, as companies are turning to technological advancement" [1]. The growing importance of external technological sources is due to the shortening of life cycle of technology, the development of emerging technologies, of cost-sharing practices and of associated risks with science based technology. Cooperation between firms regarding learning and innovation is found more often lately, mostly because of fast technology-based changes in the business world. The

\footnotetext{
*Corresponding author: cristina.feniser@mis.utcluj.ro
} 
quick rise of technological knowledge along with growing costs of R\&D lead to an improbability that any companies could keep all their production capabilities and knowledge in-house. Increasing specialization also facilitates situations in which companies occupy small positions in the space of knowledge.

In small and medium enterprises innovation becomes a business strategy in the moment when the product is a generic one, when the difference between the competition is only in the price. Such a strategy is necessary now more than ever due to technology complexity and of the shortening life span of products. Thus, in order to differentiate and to create value in a market that puts pressure on the existing products, companies choose innovation as a strategy.

Lately, the concept of capacity of absorption is more and more utilized in explaining a wide range of phenomena, from technology transfer in between states to the efficiency of international alliances. At an organizational level the term is used to examine the process of innovation and the result of accumulating knowledge within the organization to deliver a sustainable and competitive advantage. The capacity of absorption appeared as a concept in the $80 \mathrm{~s}$ when different studies underlined the base role of gaining and applying recent knowledge in order to obtain competitiveness. The term was used by Kedia and Bhagat (1998) in the setting of technology transfer in between countries and they related it with the companies' receptivity to the evolution of technology [2]. Cohen and Leginthal are the first authors to have given a concise definition and have constructed a more general theoretical framework for the application of the capacity of absorption in the business domain. The definition they gave is "the company's ability to recognize the value of a new external information, its assimilation and application in commercial purposes" [3]. (Cohen and Levinthal, 1990). This definition includes some very essential aspects. The first is the multidimensional aspect of the notion, including three fundamental capacities related to the newly discovered knowledge, namely: recognizing the value, assimilation and application. The second aspect refers to the connection between the capacity of absorption and the previous knowledge which implies certain abilities and a common language, something that shows the cumulative character of the capacity of absorption in that its present development will allow for a more efficient accumulation in the future.

Zahra and George (2002) expanded the notion of capacity of absorption through distinguishing between potential and realized capacity of absorption. The first one requires acquiring and assimilating knowledge transfers and the second one helps its application in the development and refinement of routines that help transformation and exploitation [4].

The capacity of absorption is a functional concept and it implies different capabilities depending on the company and its domain of activity. But the main elements that make the presence of the capacity of absorption possible are:

- Basic knowledge: which offers the capacity of identification, communication, understanding and evaluation of new knowledge and information;

- The organization and internal processes which motivates, coordinates and organizes people and resources, and distributes and integrates knowledge;

- The connections and relational mechanism with the external environment are those that create communication channels and collaborating relationships;

- Strategies which offers direction, coherence and lead to the acquisition and application of knowledge.

Literature dedicated to this theme highlights the increasing role of knowledge and learning in companies and it recognizes the changes that take place in generating, distributing and application patterns of knowledge in the industrial domain. After analyzing this literature Lane and Lubatkin (1998) revises the definition of the capacity of absorption and defines it as: a company's ability to utilize external knowledge using three processes: 
- Explorational learning: recognizing and understanding the knowledge of the outside environment that has potential to add value to the company.

- Transformational learning: the assimilation of valuable knowledge,

- Exploitational learning: using the knowledge assimilated in creating new knowledge and commercial results [5].

\section{Methods}

This study is based on a survey research focusing on the idea of individual perception of managers on open innovation in small and medium enterprises and was obtained by applying the research to the managers of 118 companies that were in the Top Companies in the County of Alba, who in a previous research have declared that they have implemented open innovation programs in the companies' activities they lead. Due to the nature of the problem of a questionnaire, the questionnaire was conceived for auto-application, in order to remove the effect of influence by the presence of an operator and to diminish the effect of interaction needed with one. Another reason why we applied this type of research was the fact that auto-administration implies time saving and less resources. The data was collected through e-mail and telephone.

\section{Results}

After analyzing the obtained responses, the most important reasons why managers choose to get involved in open innovation projects were identified, which are: collection of new development ideas, a better understanding of the clients' needs and expectations and access to technologies and new technical knowledge used in industry.

The advantages perceived by managers due to open innovation projects are:

- Finding new and innovating ideas;

- The development of new and innovating products and services;

- Reducing costs and risks sharing for R\&D;

- Entering new markets;

- Increasing reputation;

- Shortening the length of time to enter the market.

Based on the elements that compose the capacity of absorption identified in revised literature, we have identified five determinative factors in SMEs' activity:

- The managers' and employees' experience, both in the activity that the company carries such as open innovation projects. Most research that studied the capacity of absorption have highlighted the importance of human, educational and experience resource.

- The technology used: considered as another indicator of the capacity of absorption due to the equipment used, its level of performance is related to the achievement of complex products;

- Organizational capabilities: they are key elements in competition of SMEs;

- Activities of learning and innovation: research/development activities are a common indicator of the capacity of absorption;

- The connections with entities from the external environment.

The managers were asked which mechanisms they search for opportunities to improve technological capabilities. The mains sources of innovation come from the supplier, $75 \%$ of the respondents use this mechanism, followed by clients, for $68 \%$ of the cases included in the analysis and the internet has a frequency of $62 \%$ in the total obtained responses. The connections with researching organizations have a significant proportion of $29 \%$ in the 
economical responses. The least used sources of innovation by managers are consultants $(6 \%)$ and industrial or trade associations $(14 \%)$.

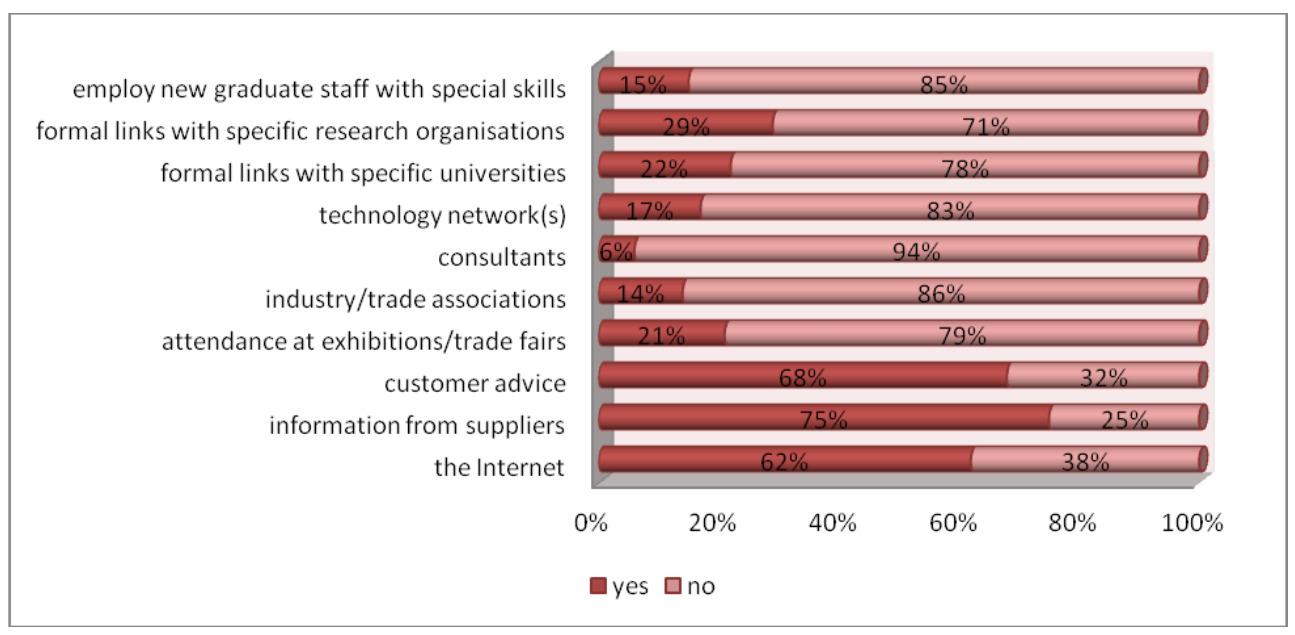

Fig. 1. Mechanisms used to identify technological and non-technological opportunities

Figure 2 illustrates which contributors are the main one for SMEs which are included in the analysis. Just as the variable analyzed previously suggests, referring to the mechanisms of identifying opportunities, the managers indicate as their main partners in innovation projects their suppliers, their clients and universities or other centers of research. This result is due to the smaller costs that this type of partnership implies, a significant aspect in the case of SMEs with a smaller budget for this type of projects.

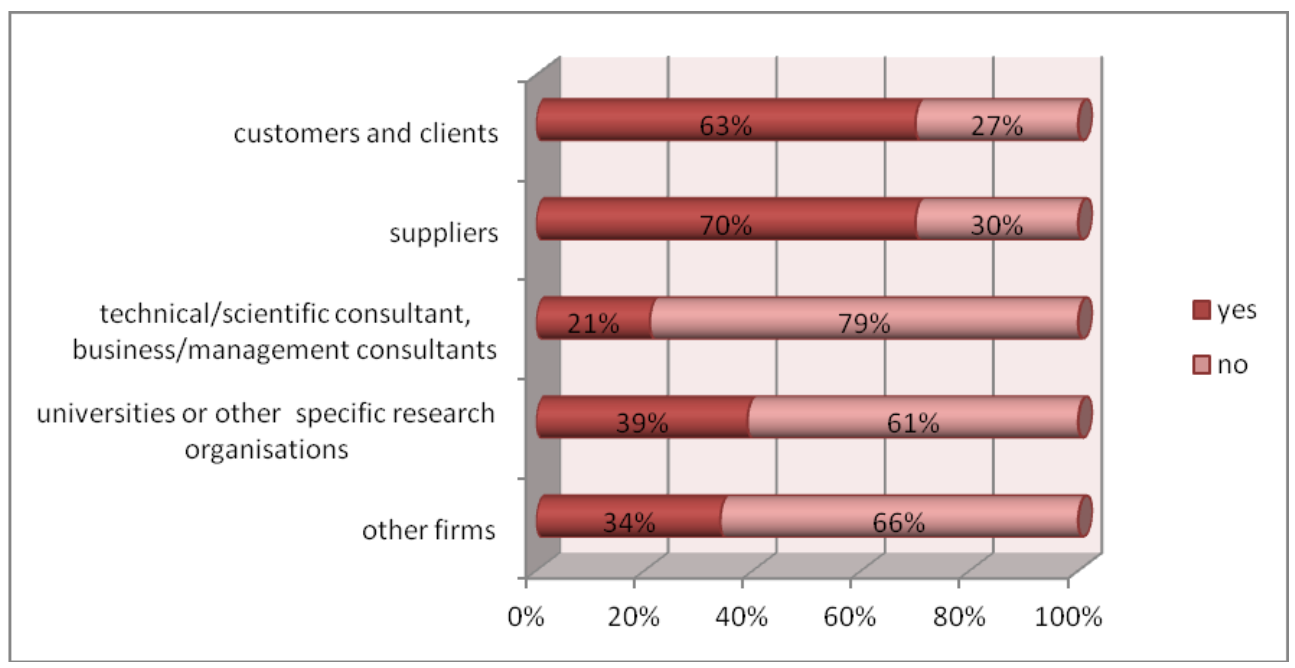

Fig. 2. Does the firm cooperate with other organizations?

\section{Conclusions}

Absorptive capacity and innovation are interconnected, in a relationship of interdependence and not as a substitute relationship. Absorptive capacity is of utmost importance in the analysis of the success some firm have had regarding others, namely relating to value creation and attraction of exterior sources, like adopting new technologies partnerships with 
others for innovation. An open innovation project doesn't end once a new product has been launched, this is just the beginning. The companies involved acquire new abilities and, thus, become more competent and more profitable. These types of initiatives always generate new opportunities, and these are finally translated in the improvement of the capacity of absorption. On the other hand, the market is very dynamic and a new product can be taken by competition in a relatively short time and new strategies always have to be taken into account.

The competition of SMEs doesn't solely depend on the employers' skills, but also on the relationship capital that the company disposes of because this strengthens the negotiation position and makes the company vigilant towards other companies of the same domain.

Through a research conducted on the companies that were the Top Companies in the County of Alba in 2010 according to the Chamber of Commerce, it shows that innovation on products and services is a strategical priority after productiveness and profit. One of the research's conclusions was that for small and medium enterprises the implementation of this type of activity greatly depends on the owner, his knowledge and abilities, on his experience and on his value system. Among the advantages identified by managers in open innovation projects are: the development of new and innovating products and services; reducing costs and risk sharing for $\mathrm{R} \& \mathrm{D}$; access to new markets; improving the company's image.

We chose to deepen the managerial perspective on open innovation and the capacity of absorption of companies in order to understand how the perceptions and decisions of a manager can improve the capacity of a firm for learning from exterior sources. In the future, open innovation is thought to be integrated in the management of innovation, this being the reason we view it as of utmost importance for the deepening of the managerial perspective regarding its influences and component elements.

The rise of economic value, besides aiding new technologies development through innovation, also means an efficient use and broadcast. The capacity of absorbing and utilizing exterior knowledge is of utmost importance for SMEs. The fact that exterior knowledge from others exists is insufficient. The company needs to be able to absorb and implement new technologies. The capacity of absorption is an important dimension for the capacity of innovation which is responsible for the identification, evaluation, acquiring and integration of external knowledge - and it has a relevance only in a general strategy and capacity for innovation. The changes that take place once globalization happens, which require a specialized knowledge based on economy increase the importance of this dimension of innovation.

The research is based on a survey regarding the topic of individual perception of managers on open innovation in small and medium enterprises and was obtained by applying the research to the managers of 118 companies that were in the Top Companies in Alba County, who in a previous research have declared that they have implemented open innovation programs in the companies' activities they lead. Due to the nature of the problem of a questionnaire, the survey was conceived for auto-application, in order to remove the effect of influence by the presence of an operator and to diminish the effect interaction needed with one. Another reason why we applied this type of research was the fact that auto-administration implies time saving and less resources. The data was collected through e-mail and telephone.

\section{References}

1. H. Chesbrough, Open innovation; The new imperative for creating and profiting from technology (Harvard Business School Press, Boston, 2003)

2. B. L. Kedia, R.S. Bhagat, Acad. Manage. Rev.,13, 559-571 (1988) 
3. W.M. Cohen, D.A. Levinthal, Admin. Sci. Q., Absorptive Capacity: a new perspective on learning and innovation, 35, 128-152 (1990)

4. S. Zahra, G. George, Acad. Manage. Rev, 27(2), 185-203 (2002)

5. P. J. Lane, M. Lubatkin, Strat. Manage. J., 19(5), 461-477 (1998) 